\title{
Correlation functions in one-dimensional spin lattices with Ising and Heisenberg bonds
}

\author{
Stefano Bellucci ${ }^{1}$ and Vadim Ohanyan ${ }^{2,3}$ \\ ${ }^{1}$ INFN-Laboratori Nazionali di Frascati, Via E. Fermi 40, 00044 Frascati, Italy \\ ${ }^{2}$ Department of Theoretical Physics, Yerevan State University, Alex Manoogian 1, 0025 Yerevan, Armenia \\ ${ }^{3}$ ICTP, Strada Costiera 11, I-34151 Trieste, Italy
}

(Dated: November 5, 2013)

\begin{abstract}
A general technique of exact calculation of any correlation functions for the special class of onedimensional spin models containing small clusters of quantum spins assembled to a chain by alternating with the single Ising spins is proposed. The technique is a natural generalization of that in the models solved by a classical transfer matrix. The general expressions for corresponding matrix operators which are the key components of the technique are obtained. As it is clear from the general principles, the decay of the correlation functions of various types is explicitly shown to be governed by a single correlation length. The technique is illustrated by two examples: symmetric diamond chain and asymmetric sawtooth chain.

PACS numbers: $75.10 . \mathrm{Jm}$

Keywords: Heisenberg-Ising chains, correlation functions, classical transfer matrix, diamond chain, sawtooth chain
\end{abstract}

\section{INTRODUCTION}

Recently, much attention has been paid to the exactly solvable models of one-dimensional magnetism, consisting of small quantum spin clusters connected to each other via Ising spins $\stackrel{1-26}{-2}$ For the sake of simplicity we will call them Heisenberg-Ising chains (HIC). The interest toward such spin systems stems from several features they possess. First of all, they allow one to obtain an exact statistical-mechanical solution of the problem of strongly interacting spins in terms of classical transfer matrix ${ }^{27-30}$. In addition to that, such systems combine the quantum and classical properties and also, to some extent, are connected to the problems and models of molecular magnetism ${ }^{31}$, as the classical transfer matrix is constructed by the diagonalization of the small quantum spin cluster. And finally, very recently the family of trimetallic coordination polymer compounds has been synthesized $32-34$. These compounds in the magnetic sense are indeed a one-dimensional magnets with both classical (Ising) and quantum (Heisenberg) bonds. The single-chain magnet reported in Ref. 32 is an example of a Heisenberg-Ising chain of triangles with three-spin linear quantum clusters; another interesting molecular magnet (spin ring) based on the highly anisotropic properties of the $\mathrm{Dy}^{3+}$ ions has been reported in Ref. 33 . Both systems have been analyzed by the transfer-matrix solution of HIC chains in Ref. 34. Another class of examples is given by chain magnets with two alternating magnetic ions, thus giving rise to alternating quantum and classical spins (see, e.g., Refs. 35 37). Let us describe the general scheme of the HIC:

- There is a small cluster of a few quantum spins with a certain topology of bonds which could contain various types of spin-spin interaction as well as interactions with external fields. The Hamiltonian of the single small cluster is, thus, a finite dimensional matrix.

- These clusters are assembled to the chain or any other one-dimensional structure by alternating with the single Ising spins.

- The interaction between the single Ising spins and any quantum spin from a small cluster must contain only $z$-components (Ising interaction).

For the mixed quantum-classical spin system with the above mentioned properties the exact solution is obtained in the following way. The idea is to apply a classical transfer-matrix formalism in which the entries of the classical transfer matrix are obtained from the diagonalization of a small quantum spin cluster. Although the number of papers devoted to the exact solutions of the $\mathrm{HIC}$ and to their magneto-thermal properties is rather impressive, only a few models have been solved within the classical transfer matrix formalism ${ }^{5,9}-11,13-17,19-22,26$, while the other method of the so-called decoration iteration transformation has been also used $1-4,8,12,24,25$. Particularly, within the direct classical transfer matrix technique the following models have been solved exactly: the double-tetrahedral chain with quantum triangles of spins 9.11 , the sawtooth chain with an asymmetric pair of quantum spins 10,13 , diamond chain $14,16,17,20-22,26$ and orthogonal-dimer chain with anisotropic and asymmetric quantum triangles 19 . Despite the complete and exact description of the ground states and ground states phase diagrams, as well of all thermodynamic functions, finitetemperature entropy and magnetization, magnetocaloric effect, etc, the expressions for the correlation function are still missing. It is worth mentioning, that the correlation functions can be in principle calculated by another method which is used to handle the HIC, the technique of the decoration iteration transformation $1-4,8,12,24,25,38-40$. However, the method of the classical transfer matrix is 
more straightforward and is based on a direct application of the canonical expectation values 41.42 , which can be calculated exactly. In the present paper we are going to develop an exact technique for the calculation of any finite-temperature correlation function for any $\mathrm{HIC}$ by means of the direct application of the classical transfermatrix method.

Beside the pure academic interest, the knowledge about the exact correlation functions of the HIC recently became important from the experimental point of view as well. As mentioned above, a few years ago it was reported about the synthesis of a novel class of trimetallic coordination polymer compounds ${ }^{32-34,36,37}$ which are the real examples of the HIC (at least one of them). As known, the neutron scattering is one of the most powerful and straightforward methods of investigation of the magnetic structure, spin textures, magnetic excitations, etc. In the leading order the cross-section of the neutron scattering is given by the two-point spin-spin correlation functions (their Fourier-transform). This fact makes the topic of the present paper a practical issue.

The paper is organized as follows. In the second Section we remind the reader of the main features of the classical transfer-matrix formalism for the HIC, in the next section we present the formalism for calculating the correlation function of $\mathrm{HIC}$ in terms of traces of certain matrix products and prove that for all kinds of correlation functions the correlation length is the same. In Section IV we apply the general formalism for several models. The paper ends with a Conclusion.

\section{CLASSICAL TRANSFER-MATRIX FORMALISM FOR HIC}

The Hamiltonian of a HIC has the form of a sum of local block Hamiltonians $\mathcal{H}_{n}$ which correspond to the n- th small quantum cluster and its interaction with left and right Ising spins:

$$
\mathcal{H}=\sum_{n=1}^{N}\left(\mathcal{H}_{n}-\frac{H}{2}\left(\sigma_{n}+\sigma_{n+1}\right)\right)
$$

here $\sigma_{n}$ is the Ising spin situated between the n-th and $(\mathrm{n}+1)$-th small cluster and $H$ is the magnetic field acting on Ising spins. The block Hamiltonian can contain various terms with quantum and classical spin variables which are determined by the topology of the elementary block and the character of interactions. Let us denote the quantum spins by $\mathbf{S}$; then one can suppose that the block Hamiltonian depends on $\mathbf{S}, \sigma$ and may be on other classical spins $\tau_{b}$,

$$
\mathcal{H}_{n}=\mathcal{H}\left(\mathbf{S}_{n, a}, \sigma_{n}, \sigma_{n+1}, \tau_{n, b}\right)
$$

Due to the properties listed above the block Hamiltonians commute to each other,

$$
\left[\mathcal{H}_{i}, \mathcal{H}_{j}\right]=0 \text {. }
$$

This fact allows one to expand the exponential in the expression for the partition function of the model and obtain an expression which by its structure corresponds to the classical transfer matrix scheme:

$$
\begin{aligned}
\mathcal{Z}= & \operatorname{Tr}_{\mathrm{S}} \sum_{(\sigma)} \sum_{(\tau)} \exp (-\beta \mathcal{H})=\sum_{(\sigma)} \prod_{n=1}^{N} e^{\beta \frac{H}{2}\left(\sigma_{n}+\sigma_{n+1}\right)} \sum_{(\tau)} \operatorname{Tr}_{n} e^{-\beta \mathcal{H}_{n}}= \\
& \sum_{(\sigma)} \prod_{n=1}^{N} e^{\beta \frac{H}{2}\left(\sigma_{n}+\sigma_{n+1}\right)} \sum_{(\tau)} \sum_{i=1}^{L} e^{-\beta \lambda_{i}\left(\sigma_{n}, \sigma_{n+1} \mid \tau_{n, b}\right)}=\sum_{(\sigma)} \prod_{n=1}^{N} T_{\sigma_{n}, \sigma_{n+1}}=\operatorname{Tr} \mathbf{T}^{N},
\end{aligned}
$$

here the cyclic boundary conditions are supposed, $\lambda_{i}\left(\sigma_{n}, \sigma_{n+1} \mid \tau_{n, b}\right)$ are the eigenvalues of the block Hamiltonian $\mathcal{H}_{n}$ (which is supposed to be an $L$ by $L$ matrix ) depending on the values of classical spins $\tau$ and classical spins $\sigma_{n}$ which alternate with the blocks, $\operatorname{Tr}_{n}$ means the trace over the states of the $n$-th quantum block, and $\mathrm{Tr}$ stands for the trace of the 2 by 2 transfer matrix with entries $T_{\sigma_{n}, \sigma_{n+1}}$. After summation over the values of $\tau$ for each block one gets the expression depending only on $\sigma_{n}$ and $\sigma_{n+1}$ which is the classical 2 by 2 transfer matrix

$$
\mathbf{T}=\left(\begin{array}{lc}
e^{\beta \frac{H}{2}} Z_{++} & Z_{+-} \\
Z_{-+} & e^{-\beta \frac{H}{2}} Z_{--}
\end{array}\right),
$$

where $Z_{\sigma_{n}, \sigma_{n+1}}=\sum_{(\tau)} \sum_{i=1}^{L} e^{-\beta \lambda_{i}\left(\sigma_{n}, \sigma_{n+1} \mid \tau_{n, b}\right)}$ is the partition function of one block, depending on the values 
of two neighboring Ising spins, signs " +" and " -" in indexes correspond to $\sigma=1 / 2$ and $\sigma=-1 / 2$ respectively. The formalism of classical transfer-matrix allows one to obtain exact analytic expressions for almost any expectation values of the function of local variables (spins) and, of course, for any thermodynamic functions, as the expression for the free energy per block can be obtained in a straightforward way by finding the maximal eigenvalue of the transfer matrix (2.5):

$$
f=-\frac{1}{\beta} \log \frac{1}{2}\left(e^{\beta \frac{H}{2}} Z_{++}+e^{-\beta \frac{H}{2}} Z_{--}+\sqrt{\left(e^{\beta \frac{H}{2}} Z_{++}-e^{-\beta \frac{H}{2}} Z_{--}\right)^{2}+4 Z_{+-} Z_{-+}}\right) .
$$

From this expression any thermodynamic function, like magnetization, entropy, specific head etc can be obtained exactly. However, to obtain correlation functions of the spins demands more efforts and further development of the classical transfer-matrix technique.

\section{CORRELATION FUNCTIONS AND CORRELATION LENGTH}

Let us concentrate on the scheme of calculation of the expectation values of combinations of spins operators. First of all, let us start from the simplest case, the expectation values of $\sigma-\mathrm{s}$, which is quite similar to that of the Ising chain 27 . Thus, for the thermodynamic average of just one $\sigma$ one obtains

$$
\begin{aligned}
\left\langle\sigma_{j}\right\rangle & =\frac{1}{\mathcal{Z}} \sum_{(\sigma)} \sigma_{j} \prod_{n=1}^{N} T_{\sigma_{n}, \sigma_{n+1}} \\
& =\frac{1}{\mathcal{Z}} \sum_{(\sigma)} T_{\sigma_{1}, \sigma_{2}} \ldots \sigma_{j} T_{\sigma_{j}, \sigma_{j+1}} \ldots T_{\sigma_{N}, \sigma_{1}}
\end{aligned}
$$

This expression is the trace of the product of $N$ transfermatrices with the matrix

$$
\sigma^{z}=\left(\begin{array}{cc}
1 / 2 & 0 \\
0 & -1 / 2
\end{array}\right)
$$

inserted before the $j$-th transfer matrix in the trace formula. Thus, we have

$$
\left\langle\sigma_{j}\right\rangle=\frac{1}{\mathcal{Z}} \operatorname{Tr}\left(\sigma^{z} \mathbf{T}^{N}\right)=\frac{1}{\mathcal{Z}} \operatorname{Tr}\left(\sigma^{z} \mathbf{A} \mathbf{\Lambda}^{N} \mathbf{A}^{-1}\right)
$$

where we used the matrix identity $\mathbf{T}=\mathbf{A} \mathbf{\Lambda} \mathbf{A}^{-1}$, with the diagonal matrix $\boldsymbol{\Lambda}$ and the orthogonal matrix $\mathbf{A}$. Thus,

$$
\boldsymbol{\Lambda}=\left(\begin{array}{cc}
\lambda_{1} & 0 \\
0 & \lambda_{2}
\end{array}\right), \quad \mathbf{A}=\left(\begin{array}{cc}
\cos \phi & -\sin \phi \\
\sin \phi & \cos \phi
\end{array}\right),
$$

where $\lambda_{1,2}$ are the eigenvalues of the transfer-matrix, and $\phi$ is a parameter. Having all these notations we now can express the canonical expectation values from Eq. (3.1) in terms of the entries of the orthogonal matrix diagonalizing the transfer-matrix. Using the properties of the trace (cyclic permutation inside the trace does not change it) one can get

$$
\begin{aligned}
\left\langle\sigma_{j}\right\rangle= & \frac{1}{\mathcal{Z}} \operatorname{Tr}\left(\mathbf{A}^{-1} \sigma^{z} \mathbf{A} \mathbf{\Lambda}^{N}\right)= \\
& \frac{1}{2} \frac{(\cos 2 \phi) \lambda_{1}^{2}-(\sin 2 \phi) \lambda_{2}^{N}}{\lambda_{1}^{N}+\lambda_{2}^{N}}
\end{aligned}
$$

Now, we should take care about the thermodynamic limit $(N \rightarrow \infty)$. It is easy to see, that in this limit Eq. 3.5 leads to (suppose that $\lambda_{1}$ is greater than $\lambda_{2}$ )

$$
\left\langle\sigma_{j}\right\rangle=\frac{1}{2} \cos 2 \phi \equiv \frac{1}{2} M_{\sigma},
$$

where $M_{\sigma}$ is the partial magnetization corresponding to the subsystem with $\sigma$-spins,

$$
M_{\sigma}=\frac{e^{\beta \frac{H}{2}} Z_{++}-e^{-\beta \frac{H}{2}} Z_{--}}{\sqrt{\left(e^{\beta \frac{H}{2}} Z_{++}-e^{-\beta \frac{H}{2}} Z_{--}\right)^{2}+4 Z_{+-} Z_{-+}}}
$$

Thus, we can express the entries of the orthogonal matrix diagonalizing the transfer matrix in terms of the subsystem magnetization $M_{\sigma}$ :

$$
\mathbf{A}=\frac{1}{\sqrt{2}}\left(\begin{array}{cc}
\sqrt{1+M_{\sigma}} & -\sqrt{1-M_{\sigma}} \\
\sqrt{1-M_{\sigma}} & \sqrt{1+M_{\sigma}}
\end{array}\right)
$$

These quantities are the building blocks for the calculation of all correlation function for the HIC. As will be clear from further consideration, any correlation function of HIC has the form of the trace of a certain product of transfer matrices and special matrices representing the spin operators. Moreover, these matrices are constructed from the local expectation values of spin operators with the aid of the matrix $\mathbf{A}$ in the standard way $\widetilde{\mathbf{P}} \equiv \mathbf{A}^{-1} \mathbf{P A}$. Let us write down the entries of a general matrix of that type 


$$
\begin{aligned}
& \widetilde{P}_{++}=\frac{1}{2}\left[\left(1+M_{\sigma}\right) P_{++}+\sqrt{1-M_{\sigma}^{2}}\left(P_{+-}+P_{-+}\right)+\left(1-M_{\sigma}\right) P_{--}\right], \\
& \widetilde{P}_{+-}=\frac{1}{2}\left[\sqrt{1-M_{\sigma}^{2}}\left(P_{--}-P_{++}\right)+\left(1+M_{\sigma}\right) P_{+-}-\left(1-M_{\sigma}\right) P_{-+}\right], \\
& \widetilde{P}_{-+}=\frac{1}{2}\left[\sqrt{1-M_{\sigma}^{2}}\left(P_{--}-P_{++}\right)+\left(1+M_{\sigma}\right) P_{-+}-\left(1-M_{\sigma}\right) P_{+-}\right], \\
& \widetilde{P}_{--}=\frac{1}{2}\left[\left(1-M_{\sigma}\right) P_{++}-\sqrt{1-M_{\sigma}^{2}}\left(P_{+-}+P_{-+}\right)+\left(1+M_{\sigma}\right) P_{--}\right] .
\end{aligned}
$$

For instance, the matrix $\widetilde{\sigma}^{z}=\mathbf{A}^{-1} \sigma_{z} \mathbf{A}$ which is the building block for constructing various correlation functions of $\sigma_{j}$ has a particulary simple form:

$$
\tilde{\sigma}^{z}=\frac{1}{2}\left(\begin{array}{lc}
M_{\sigma} & -\sqrt{1-M_{\sigma}^{2}} \\
-\sqrt{1-M_{\sigma}^{2}} & -M_{\sigma}
\end{array}\right)
$$

The simple two-site correlation function is just a thermal average of the product of two $\sigma$ spins situated at a distance $r$ from each other:

$$
\left\langle\sigma_{j} \sigma_{j+r}\right\rangle=\frac{1}{\mathcal{Z}} \sum_{(\sigma)} \sigma_{j} \sigma_{j+r} \prod_{n=1}^{N} T_{\sigma_{n}, \sigma_{n+1}}=\frac{1}{\mathcal{Z}} \operatorname{Tr}\left(\mathbf{T}^{N-r} \sigma^{z} \mathbf{T}^{r} \sigma^{z}\right)=\frac{1}{\mathcal{Z}} \operatorname{Tr}\left(\boldsymbol{\Lambda}^{N-r} \widetilde{\sigma}^{z} \boldsymbol{\Lambda}^{r} \widetilde{\sigma}^{z}\right)
$$

Thus, using the matrix form of $\widetilde{\sigma}^{z}$ and taking a thermodynamic limit one obtains:

$$
\begin{aligned}
& \left\langle\sigma_{j} \sigma_{j+r}\right\rangle=\frac{1}{4}\left(M_{\sigma}^{2}+\left(1-M_{\sigma}^{2}\right) e^{-\frac{r}{\xi}}\right), \\
& \xi=\frac{1}{\log \left(\frac{\lambda_{1}}{\lambda_{2}}\right)},
\end{aligned}
$$

where $\xi$ is a correlation length. So far, all results have the same form as those for a simple Ising chain, the difference is only in the form of the magnetization for $\sigma$-spins, which in our case is given by Eqs. (3.7) and (2.5). Now, let us turn to the more complicated correlation functions of the quantum spins with each other and mixed correlation functions, 


$$
\begin{aligned}
& \left\langle S_{j, a}^{\alpha} S_{j+r, b}^{\beta}\right\rangle=\frac{1}{\mathcal{Z}} \operatorname{Tr}_{S} \sum_{(\sigma)} \sum_{(\tau)} S_{j, a}^{\alpha} S_{j+r, b}^{\beta} \prod_{n=1}^{N} e^{-\beta \mathcal{H}_{n}} e^{\beta \frac{H}{2}\left(\sigma_{n}+\sigma_{n+1}\right)}=\frac{1}{\mathcal{Z}} \operatorname{Tr}\left(\Lambda^{N-r-1} \widetilde{\boldsymbol{\Sigma}}_{a}^{\alpha} \Lambda^{r-1} \widetilde{\mathbf{\Sigma}}_{b}^{\beta}\right), \\
& \left\langle S_{j, a}^{\alpha} \sigma_{j+r}\right\rangle=\frac{1}{\mathcal{Z}} \operatorname{Tr}_{S} \sum_{(\sigma)} \sum_{(\tau)} S_{j, a}^{\alpha} \sigma_{j+r} \prod_{n=1}^{N} e^{-\beta \mathcal{H} n} e^{\beta \frac{H}{2}\left(\sigma_{n}+\sigma_{n+1}\right)}=\frac{1}{\mathcal{Z}} \operatorname{Tr}\left(\Lambda^{N-r-1} \widetilde{\boldsymbol{\Sigma}}_{a}^{\alpha} \Lambda^{r} \widetilde{\sigma}^{z}\right) \\
& \left\langle S_{j, a}^{\alpha} \tau_{j+r, b}\right\rangle=\frac{1}{\mathcal{Z}} \operatorname{Tr}_{S} \sum_{(\sigma)} \sum_{(\tau)} S_{j, a}^{\alpha} \tau_{j+r, b} \prod_{n=1}^{N} e^{-\beta \mathcal{H}_{n}} e^{\beta \frac{H}{2}\left(\sigma_{n}+\sigma_{n+1}\right)}=\frac{1}{\mathcal{Z}} \operatorname{Tr}\left(\Lambda^{N-r-1} \widetilde{\boldsymbol{\Sigma}}_{a}^{\alpha} \Lambda^{r-1} \widetilde{\tau}_{b}\right), \\
& \left\langle\tau_{j, a} S_{j+r, b}^{\alpha}\right\rangle=\frac{1}{\mathcal{Z}} \operatorname{Tr}_{S} \sum_{(\sigma)} \sum_{(\tau)} \tau_{j, a} S_{j+r, b}^{\alpha} \prod_{n=1}^{N} e^{-\beta \mathcal{H}_{n}} e^{\beta \frac{H}{2}\left(\sigma_{n}+\sigma_{n+1}\right)}=\frac{1}{\mathcal{Z}} \operatorname{Tr}\left(\Lambda^{N-r-1} \widetilde{\tau}_{a} \Lambda^{r-1} \widetilde{\boldsymbol{\Sigma}}_{b}^{\alpha}\right), \\
& \left\langle\tau_{j, a} \tau_{j+r, b}\right\rangle=\frac{1}{\mathcal{Z}} \operatorname{Tr}_{S} \sum_{(\sigma)} \sum_{(\tau)} \tau_{j, a} \tau_{j+r, b} \prod_{n=1}^{N} e^{-\beta \mathcal{H}_{n}} e^{\beta \frac{H}{2}\left(\sigma_{n}+\sigma_{n+1}\right)}=\frac{1}{\mathcal{Z}} \operatorname{Tr}\left(\Lambda^{N-r-1} \widetilde{\tau}_{a} \Lambda^{r-1} \widetilde{\tau}_{b}\right), \\
& \left\langle\tau_{j, a} \sigma_{j+r}\right\rangle=\frac{1}{\mathcal{Z}} \operatorname{Tr}_{S} \sum_{(\sigma)} \sum_{(\tau)} \tau_{j, a} \sigma_{j+r} \prod_{n=1}^{N} e^{-\beta \mathcal{H}} e^{\beta \frac{H}{2}\left(\sigma_{n}+\sigma_{n+1}\right)}=\frac{1}{\mathcal{Z}} \operatorname{Tr}\left(\Lambda^{N-r-1} \widetilde{\tau}_{a} \Lambda^{r} \widetilde{\sigma}\right), \\
& \left\langle\sigma_{j} S_{j+r, a}^{\alpha}\right\rangle=\frac{1}{\mathcal{Z}} \operatorname{Tr}_{S} \sum_{(\sigma)} \sum_{(\tau)} \sigma_{j} S_{j+r, a}^{\alpha} \prod_{n=1}^{N} e^{-\beta \mathcal{H}} e^{\beta \frac{H}{2}\left(\sigma_{n}+\sigma_{n+1}\right)}=\frac{1}{\mathcal{Z}} \operatorname{Tr}\left(\Lambda^{N-r} \widetilde{\sigma}^{z} \Lambda^{r-1} \widetilde{\boldsymbol{\Sigma}}_{a}^{\alpha}\right) \\
& \left\langle\sigma_{j} \tau_{j+r, a}\right\rangle=\frac{1}{\mathcal{Z}} \operatorname{Tr}_{S} \sum_{(\sigma)} \sum_{(\tau)} \sigma_{j} \tau_{j+r, a} \prod_{n=1}^{N} e^{-\beta \mathcal{H}_{n}} e^{\beta \frac{H}{2}\left(\sigma_{n}+\sigma_{n+1}\right)}=\frac{1}{\mathcal{Z}} \operatorname{Tr}\left(\Lambda^{N-r} \widetilde{\sigma} \Lambda^{r-1} \widetilde{\tau}_{a}\right),
\end{aligned}
$$

where the new 2 by 2 matrices $\boldsymbol{\Sigma}_{a}^{\alpha}$ and $\tau_{a}$ are the classical transfer-matrix formalism representation of the $\alpha$ component of the $a$-th quantum spin and classical $a$-th spins from the corresponding quantum clusters. Their entries are found according to

$$
\begin{aligned}
& \left(\Sigma_{a}^{\alpha}\right)_{\sigma_{j}, \sigma_{j+1}}=e^{\beta \frac{H}{2}\left(\sigma_{j}+\sigma_{j+1}\right)} \sum_{\left(\tau_{j}\right)} \operatorname{Tr}_{j}\left(S_{j, a}^{\alpha} e^{-\beta \mathcal{H}_{j}}\right) \\
& \left(\tau_{a}\right)_{\sigma_{j}, \sigma_{j+1}}=e^{\beta \frac{H}{2}\left(\sigma_{j}+\sigma_{j+1}\right)} \sum_{\left(\tau_{j}\right)} \tau_{j, a} \operatorname{Tr}_{j} e^{-\beta \mathcal{H}_{j}}
\end{aligned}
$$

The correlation functions containing the quantum spins $S$ have the unusual overall power of the $\boldsymbol{\Lambda}$-matrix smaller than the number of the blocks $N$. The point is that the inclusion of each quantum spin into the function reduces the overall power of the $\boldsymbol{\Lambda}$-matrix by one. This technical difference from the standard classical technique $\underline{41.42}$ stems out from the following feature. One does not have the spin operators $\mathbf{S}$ by themselves under the trace in the canonical expectation value expression corresponding to the correlation function. Instead, there are some complicated matrices given by Eq. (3.13) and representing the quantum spin operators in the generalized classical transfer-matrix formalism. As it is seen from Eq. (3.13), due to the quantum nature of the variables $S$, the corresponding matrix representation for them adsorbs the transfer matrix and it is an issue to find a representation where the resulting matrix under the trace into the partition function would be just a product of the transfermatrix and some other matrix. On the contrary, for the classical spin chains for arbitrary spin the expression $S_{j} T_{S_{j}, S_{j+1}}$ is the analog of the Eq. (3.13) which appears under the trace formula in the corresponding correlation function. Thus, the corresponding matrix to be inserted into the trace formula can be easily and naturally split into the product of the transfer matrix and a diagonal matrix with the possible values of the classical variable $S$ on its diagonal27,41,42. This fact leads also to another consequence concerning the properties of the correlation functions from Eq. (3.12). The expressions are no longer valid for $r=0$. All these cases should be considered separately out of the framework of the technique developed here. However, the correlation functions at $r=0$ for the same spin operator are trivial because they are just a expectation values of the square of spin, while correlation functions for the different spins belonging to the same block can be easily calculated by taking a derivatives of the free energy with respect to the corresponding coupling constants.

As the expressions under the trace in Eq.(3.12) have the same structure, let us write down a general expression for such a correlation function

$$
\begin{aligned}
& \frac{1}{\mathcal{Z}} \operatorname{Tr}\left(\Lambda^{N-r-1} \widetilde{\mathbf{P}} \Lambda^{r-1} \widetilde{\mathbf{R}}\right) \\
& =\frac{1}{\lambda_{1}^{2}} \widetilde{P}_{++} \widetilde{R}_{++}+\frac{1}{\lambda_{1} \lambda_{2}} \widetilde{P}_{+-} \widetilde{R}_{-+} e^{-r / \xi}
\end{aligned}
$$


where the correlation length $\xi$ is the same as in Eq. (3.11). Obviously, the part independent of $r$ is the product of expectation values of quantities corresponding to matrices $\mathbf{P}$ and $\mathbf{R}$,

$$
\left\langle P_{j}\right\rangle=\frac{1}{\mathcal{Z}} \operatorname{Tr}\left(\Lambda^{N-1} \widetilde{\mathbf{P}}\right)=\frac{1}{\lambda_{1}} \widetilde{P}_{++} .
$$

The product of two eigenvalues of the 2 by 2 transfer matrix is its determinant, thus, eventually one can write down the following general form of pair-correlation function for HIC:

$$
\begin{aligned}
& \left\langle P_{j} R_{j+r}\right\rangle=\frac{1}{\mathcal{Z}} \operatorname{Tr}\left(\Lambda^{N-r-1} \widetilde{\mathbf{P}} \Lambda^{r-1} \widetilde{\mathbf{R}}\right) \\
& =\left\langle P_{j}\right\rangle\left\langle R_{j}\right\rangle+\frac{\widetilde{P}_{+-} \widetilde{R}_{-+}}{\operatorname{det} \mathbf{T}} e^{-r / \xi}
\end{aligned}
$$

If one of the matrices is $\sigma$ the result is different, because the corresponding expression contains one more transfer matrix. In general, it also depends on the position of the $\sigma$, unless the matrix $\widetilde{\mathbf{P}}$ is symmetric. Thus, the corresponding expressions for the correlation functions are

$$
\begin{aligned}
& \frac{1}{\mathcal{Z}} \operatorname{Tr}\left(\Lambda^{N-r-1} \widetilde{\mathbf{P}} \Lambda^{r} \widetilde{\sigma}\right) \\
& =\left\langle P_{j}\right\rangle\left\langle\sigma_{j}\right\rangle-\frac{\widetilde{P}_{+-} \sqrt{1-M_{\sigma}^{2}}}{2 \lambda_{1}} e^{-r / \xi}, \\
& \frac{1}{\mathcal{Z}} \operatorname{Tr}\left(\Lambda^{N-r} \widetilde{\sigma} \Lambda^{r-1} \widetilde{\mathbf{P}}\right) \\
& =\left\langle P_{j}\right\rangle\left\langle\sigma_{j}\right\rangle-\frac{\widetilde{P}_{-+} \sqrt{1-M_{\sigma}^{2}}}{2 \lambda_{1}} e^{-r / \xi} .
\end{aligned}
$$

The matrix $\widetilde{\mathbf{P}}$, in its turn, is symmetric when the corresponding matrix $\mathbf{P}$ is symmetric. Thus, the order of $P_{j}$ and $\sigma_{i}$ in the correlation function does not matter in this case: $\left\langle P_{j} \sigma_{j+r}\right\rangle=\left\langle\sigma_{j} P_{j+r}\right\rangle$. From this general consideration one can see that there exists only a single correlation length for any kind of correlation functions of the HIC. This is rather expected result because the appearance of a single correlation length even in the inhomogeneous situation is the common feature of all one-dimensional systems which do not become critical at finite temperature. This is, however, true only for a long range correlation with respect to the block structure of the system; inside one block short range correlations are non-uniform.

\section{EXAMPLES}

In this section we are going to illustrate the technique of calculation of correlation functions for HIC by several examples. As the technique, despite its clearness and simplicity, leads to rather cumbersome expressions, we will present explicit calculations for the simplest models which have been investigated earlier in a context of magnetic and thermodynamic properties $\underline{1-26}$.



FIG. 1: Heisenberg-Ising diamond chain. Solid (dashed) lines denote quantum XXZ (classical Ising) interaction bonds. All dashed bonds correspond to the coupling constant $K$, the interaction between quantum spins is characterized by the coupling constant $J$ and anisotropy $\Delta$.

One can distinguish at least two classes of HIC: the models where each block has a left-right symmetry with respect to the interaction with $\sigma$ spins $\underline{\underline{1}-9,11,14}$, which implies that the term describing the interaction between block spins and their left and right $\sigma$-neighbors has the form $K\left(\sigma_{j}+\sigma_{j+1}\right) \sum_{a} S_{j, a}^{z}$, which is completely symmetric with respect to the permutation of the operators $S_{j, a}^{z}$, and the models which are non symmetric with respect to left and right $\sigma$-spins on each block ${ }^{10,13,19}$. It is worth mentioning, that the experimentally obtained single chain magnet compound with Ising and Heisenberg bonds, which is an existing example of HIC, belongs to the second type 32,34 . Magnetic properties of the asymmetric model are vast and complicated, they can exhibit a large number of ground states with a breaking of translational symmetry or doubling of the unit cell (block) $\underline{10,13,19}$. In the most symmetric case the block Hamiltonian is completely symmetric with respect to the permutation of all spins in it. Such models are the simplest ones. For instance, the various variants of the Heisenberg-Ising diamond chain $4,8,14$ and HeisenebrgIsing tetrahedral ${ }^{\underline{5}}$ and double-tetrahedral chains $\underline{9}, 11$ belong to this class of HIC. It is obvious, that in this case the matrices $\boldsymbol{\Sigma}_{\mathbf{a}}^{\alpha}$ are the same for all spins inside one block, or, in other words, in this case one does not need the additional index $a$.

\section{A. Symmetric diamond-chain}

The symmetric Heisenberg-Ising diamond chain is the simplest example of HIC (See Figure 1). Its block Hamiltonian has the form:

$$
\begin{aligned}
& \mathcal{H}_{n}^{D C}=J\left(\Delta\left(S_{n, 1}^{x} S_{n, 2}^{x}+S_{n, 1}^{y} S_{n, 2}^{y}\right)+S_{n, 1}^{z} S_{n, 2}^{z}\right) \\
& -\left(H-K\left(\sigma_{n}+\sigma_{n+1}\right)\right)\left(S_{n, 1}^{z}+S_{n, 2}^{z}\right)
\end{aligned}
$$

The corresponding transfer-matrix is 


$$
\mathbf{T}^{D C}=2 e^{-\beta \frac{J}{4}}\left(\begin{array}{lc}
e^{\beta \frac{H}{2}}\left(\operatorname{ch}[\beta(H-K)]+Q^{D C}\right) & \operatorname{ch}(\beta H)+Q^{D C} \\
\operatorname{ch}(\beta H)+Q^{D C} & e^{-\beta \frac{H}{2}}\left(\operatorname{ch}[\beta(H+K)]+Q^{D C}\right)
\end{array}\right),
$$

where

$$
Q^{D C}=e^{\beta \frac{J}{2}} \operatorname{ch}\left(\beta \frac{J \Delta}{2}\right)
$$

As the block Hamiltonian is $\mathrm{SO}(2)$-invariant with respect to the $z$-axis the corresponding $\boldsymbol{\Sigma}_{a}^{\alpha}$-matrix is non-zero only for $\alpha=z$ and, as discussed above, in virtue of the symmetry with respect to permutation of $\mathbf{S}$-operators for one block $\boldsymbol{\Sigma}_{1}^{z}=\boldsymbol{\Sigma}_{2}^{z} \equiv \boldsymbol{\Sigma}^{z}$. The matrix $\boldsymbol{\Sigma}^{z}$ has a rather simple form:

$$
\begin{aligned}
& \boldsymbol{\Sigma}^{z}= \\
& e^{-\beta \frac{J}{4}}\left(\begin{array}{lc}
e^{\beta \frac{H}{2}} \operatorname{sh}[\beta(H-K)] & \operatorname{sh}(\beta H) \\
\operatorname{sh}(\beta H) & e^{-\beta \frac{H}{2}} \operatorname{sh}[\beta(H+K)]
\end{array}\right)
\end{aligned}
$$

According to Eqs.(3.9)-(3.17), the non-zero two-site correlation functions for this model are

$$
\begin{aligned}
& \left\langle S_{j, a}^{z} S_{j+r, b}^{z}\right\rangle-\left\langle S_{j, a}^{z}\right\rangle^{2}=\frac{\left(2 \operatorname{sh}(\beta H) M_{\sigma}+\left[e^{\beta K} \operatorname{ch}\left(\beta \frac{H}{2}\right)-e^{-\beta K} \operatorname{ch}\left(\beta \frac{3 H}{2}\right)\right] \sqrt{1-M_{\sigma}^{2}}\right)^{2}}{16\left(\operatorname{sh}^{2}(\beta K)+2 Q^{D C}(\operatorname{ch}(\beta K)-1) \operatorname{ch}(\beta H)\right)} e^{-\frac{r}{\xi}} \\
& \left\langle S_{j, a}^{z} \sigma_{j+r}^{z}\right\rangle-\left\langle S_{j, a}^{z}\right\rangle\left\langle\sigma_{j}\right\rangle=-\frac{e^{-\beta \frac{J}{4}}\left\{2 \operatorname{sh}(\beta H) M_{\sigma} \sqrt{1-M_{\sigma}^{2}}+\left[e^{\beta K} \operatorname{ch}\left(\beta \frac{H}{2}\right)-e^{-\beta K} \operatorname{ch}\left(\beta \frac{3 H}{2}\right)\right]\left(1-M_{\sigma}^{2}\right)^{2}\right\}}{4 \lambda_{1}^{D C}} e^{-\frac{r}{\xi}}
\end{aligned}
$$

where

$$
\left\langle S_{j, a}^{z}\right\rangle=\frac{1}{\mathcal{Z}} \operatorname{Tr}\left(\Lambda^{N-1} \widetilde{\mathbf{\Sigma}^{\mathbf{z}}}\right)=\frac{1}{2} M_{S}
$$

is the partial magnetization of the sublattice of quantum spins. $M_{\sigma}$ is the magnetization of $\sigma$-sublattice obtained according to Eq.(3.7) from transfer-matrix (4.2) and $\lambda_{1}^{D C}$ is the largest eigenvalue of the transfer-matrix (4.2). The eigenvalues have the following form:

$$
\begin{aligned}
\lambda_{1,2}^{D C}=e^{-\beta \frac{J}{4}} & \left\{e^{-\beta K} \operatorname{ch}\left(\beta \frac{3 H}{2}\right)+\left(2 Q^{D C}+e^{\beta K}\right) \operatorname{ch}\left(\beta \frac{H}{2}\right)\right. \\
& \left. \pm \sqrt{\left[e^{-\beta K} \operatorname{sh}\left(\beta \frac{3 H}{2}\right)+\left(2 Q^{D C}-e^{\beta K}\right) \operatorname{sh}\left(\beta \frac{H}{2}\right)\right]^{2}+4\left(\operatorname{ch}(\beta H)+Q^{D C}\right)^{2}}\right\} .
\end{aligned}
$$

\section{B. Asymmetric sawtooth chain}

The next example we are going to consider is the Heisenberg-Ising sawtooth chain introduced in Refs. 10 and 13. The lattice is depicted in Figure 2. This model is quite convenient for illustrating the asymmetric role of quantum spins from one block as well as for the demonstration of the role of the additional classical spin included in the block. Here the block Hamiltonian contains two parts, one with quantum operators and another one
- without them. Thus, one can write down the block Hamiltonian for the sawtooth HIC with 2 quantum spins in one block in the following way:

$$
\begin{aligned}
& \mathcal{H}_{n}^{S C}=\mathcal{H}_{n}^{q}+K \sigma_{n} \tau_{n}-H \tau_{n} \\
& \mathcal{H}_{n}^{q}=J\left(\Delta\left(S_{n, 1}^{x} S_{n, 2}^{x}+S_{n, 1}^{y} S_{n, 2}^{y}\right)+S_{n, 1}^{z} S_{n, 2}^{z}\right) \\
& -\left(H-K\left(\sigma_{n}+\sigma_{n+1}+\tau_{n}\right)\right) S_{n, 1}^{z}-\left(H-K \sigma_{n+1}\right) S_{n, 2}^{z} .
\end{aligned}
$$

Here all notations are explained in Figure 2 In contrast to the case considered previously in Refs. 10 and 


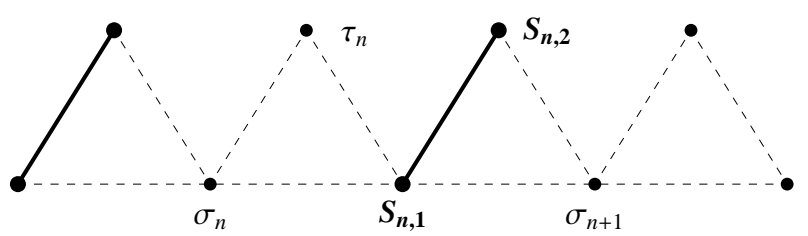

FIG. 2: Heisenberg-Ising sawtooth chain with a pair of quantum spins in one block. The unit cell consists of 4 spins, $\mathbf{S}_{j, 1}, \mathbf{S}_{j, 2}, \sigma_{j}$ and $\tau_{j}$. All bonds denoted by dashed lines are of Ising type and the corresponding coupling constant is $K$, while the $X X Z$-interaction corresponding to the quantum bond (solid line) is given by the coupling and anisotropy $J$ and $\Delta$.
13, here, for the sake of simplicity, we consider all Ising bonds with the same coupling constant $K$. As the block Hamiltonian contains also the Ising spin $\tau$, in order to obtain the entries of transfer matrix one needs, after taking the trace over quantum states of the pair of $\mathbf{S}$-spin, to sum over the two possible values of the classical spin $\tau$. The entries of the resulting classical transfer matrix are:

$$
\begin{aligned}
& T_{++}^{S C}=2 e^{-\beta \frac{J}{4}}\left\{e^{\beta\left(H-\frac{K}{4}\right)}\left[\operatorname{ch}(\beta(H-K))+Q_{2}^{S C}\right]+e^{\beta \frac{K}{4}}\left[\operatorname{ch}\left(\beta\left(H-\frac{K}{2}\right)\right)+Q_{1}^{S C}\right]\right\}, \\
& T_{+-}^{S C}=2 e^{-\beta \frac{J}{4}}\left\{e^{\frac{\beta}{2}\left(H-\frac{K}{2}\right)}\left[\operatorname{ch}(\beta H)+Q_{2}^{S C}\right]+e^{-\frac{\beta}{2}\left(H-\frac{K}{2}\right)}\left[\operatorname{ch}\left(\beta\left(H+\frac{K}{2}\right)\right)+Q_{1}^{S C}\right]\right\}, \\
& T_{-+}^{S C}=2 e^{-\frac{\beta}{4}}\left\{e^{-\frac{\beta}{2}\left(H+\frac{K}{2}\right)}\left[\operatorname{ch}(\beta H)+Q_{2}^{S C}\right]+e^{\frac{\beta}{2}\left(H+\frac{K}{2}\right)}\left[\operatorname{ch}\left(\beta\left(H-\frac{K}{2}\right)\right)+Q_{1}^{S C}\right]\right\}, \\
& T_{--}^{S C}=2 e^{-\beta \frac{J}{4}}\left\{e^{-\beta\left(H+\frac{K}{4}\right)}\left[\operatorname{ch}(\beta(H+K))+Q_{2}^{S C}\right]+e^{\beta \frac{K}{4}}\left[\operatorname{ch}\left(\beta\left(H+\frac{K}{2}\right)\right)+Q_{1}^{S C}\right]\right\},
\end{aligned}
$$

where

$$
\begin{aligned}
& Q_{1}^{S C}=e^{\beta \frac{J}{2}} \operatorname{ch}\left(\beta \frac{J \Delta}{2}\right), \\
& Q_{2}^{S C}=e^{\beta \frac{J}{2}} \operatorname{ch}\left(\beta \frac{\sqrt{K^{2}+J^{2} \Delta^{2}}}{2}\right) .
\end{aligned}
$$

The block Hamiltonian (4.8) is not symmetric with respect the to permutation of the quantum spin operators
$\mathbf{S}_{n, 1}$ and $\mathbf{S}_{n, 2}$, thus, there are two matrices representing the $z$-component of the corresponding spin-operators using for the calculation of the correlation functions. In virtue of the $\mathrm{SO}(2)$-invariance of Eq.(4.8) the corresponding matrices for other spin components are identically equal to zero. Let us write down the entries of the $\boldsymbol{\Sigma}_{1}^{z}$ and $\boldsymbol{\Sigma}_{2}^{z}$ matrices for the Heisenberg-Ising sawtooth chain.

$$
\begin{aligned}
& \left(\boldsymbol{\Sigma}_{1,2}^{z}\right)_{++}=e^{-\beta \frac{J}{4}}\left\{e^{\beta \frac{K}{4}} \operatorname{ch}\left(\beta\left(H-\frac{K}{2}\right)\right)+e^{\beta\left(H-\frac{K}{4}\right)}(\operatorname{ch}(\beta(H-K)) \mp W)\right\}, \\
& \left(\Sigma_{1,2}^{z}\right)_{+-}=e^{-\beta \frac{J}{4}}\left\{e^{-\frac{\beta}{2}\left(H-\frac{K}{2}\right)} \operatorname{ch}\left(\beta\left(H+\frac{K}{2}\right)\right)+e^{\frac{\beta}{2}\left(H-\frac{K}{2}\right)}(\operatorname{ch}(\beta H) \mp W)\right\}, \\
& \left(\Sigma_{1,2}^{z}\right)_{-+}=e^{-\beta \frac{J}{4}}\left\{e^{\frac{\beta}{2}\left(H+\frac{K}{2}\right)} \operatorname{ch}\left(\beta\left(H-\frac{K}{2}\right)\right)+e^{-\frac{\beta}{2}\left(H+\frac{K}{2}\right)}(\operatorname{ch}(\beta H) \pm W)\right\}, \\
& \left(\Sigma_{1,2}^{z}\right)_{--}=e^{-\beta \frac{J}{4}}\left\{e^{\beta \frac{K}{4}} \operatorname{ch}\left(\beta\left(H+\frac{K}{2}\right)\right)+e^{-\beta\left(H+\frac{K}{4}\right)}(\operatorname{ch}(\beta(H+K)) \pm W)\right\},
\end{aligned}
$$

where

$$
W=K \frac{e^{\beta \frac{J}{2}} \operatorname{sh}\left(\beta \frac{\sqrt{K^{2}+J^{2} \Delta^{2}}}{2}\right)}{\sqrt{K^{2}+J^{2} \Delta^{2}}} .
$$

and upper(lower) signs correspond to $\boldsymbol{\Sigma}_{1}^{z}\left(\boldsymbol{\Sigma}_{2}^{z}\right)$. The matrix $\tau$ in terms of which the expectation values and cor- 
relation function of the classical spin from block Hamil-

tonian are expressed has the following entries:

$$
\begin{aligned}
& \tau_{++}=e^{-\beta \frac{J}{4}}\left\{e^{\beta\left(H-\frac{K}{4}\right)}\left[\operatorname{ch}(\beta(H-K))+Q_{2}^{S C}\right]-e^{\beta \frac{K}{4}}\left[\operatorname{ch}\left(\beta\left(H-\frac{K}{2}\right)\right)+Q_{1}^{S C}\right]\right\}, \\
& \tau_{+-}=e^{-\beta \frac{J}{4}}\left\{e^{\frac{\beta}{2}\left(H-\frac{K}{2}\right)}\left[\operatorname{ch}(\beta H)+Q_{2}^{S C}\right]-e^{-\frac{\beta}{2}\left(H-\frac{K}{2}\right)}\left[\operatorname{ch}\left(\beta\left(H+\frac{K}{2}\right)\right)+Q_{1}^{S C}\right]\right\}, \\
& \tau_{-+}=e^{-\frac{\beta}{4}}\left\{-e^{-\frac{\beta}{2}\left(H+\frac{K}{2}\right)}\left[\operatorname{ch}(\beta H)+Q_{2}^{S C}\right]+e^{\frac{\beta}{2}\left(H+\frac{K}{2}\right)}\left[\operatorname{ch}\left(\beta\left(H-\frac{K}{2}\right)\right)+Q_{1}^{S C}\right]\right\}, \\
& \tau_{--}=e^{-\beta \frac{J}{4}}\left\{-e^{-\beta\left(H+\frac{K}{4}\right)}\left[\operatorname{ch}(\beta(H+K))+Q_{2}^{S C}\right]+e^{\beta \frac{K}{4}}\left[\operatorname{ch}\left(\beta\left(H+\frac{K}{2}\right)\right)+Q_{1}^{S C}\right]\right\} .
\end{aligned}
$$

All possible two-spin correlation functions (except the $\sigma \sigma$, which is uniform for all HIC and is given by Eq.
(3.12) of the system are listed below, 


$$
\begin{aligned}
& \left\langle S_{j, 1}^{z} S_{j+r, 1}^{z}\right\rangle-\left\langle S_{j, 1}^{z}\right\rangle^{2}=\frac{\left(\sum_{k=1}^{4}\left[A_{k}^{1} \operatorname{sh}\left(\beta \frac{k H}{2}\right)+B_{k}^{1} \operatorname{ch}\left(\beta \frac{k H}{2}\right)\right]\right)\left(\sum_{k=1}^{4}\left[A_{k}^{3} \operatorname{sh}\left(\beta \frac{k H}{2}\right)+B_{k}^{3} \operatorname{ch}\left(\beta \frac{k H}{2}\right)\right]\right)}{16 \sum_{k=0}^{2} D_{k} \operatorname{ch}(\beta k H)} e^{-\frac{r}{\xi}}, \\
& \left\langle S_{j, 2}^{z} S_{j+r, 2}^{z}\right\rangle-\left\langle S_{j, 2}^{z}\right\rangle^{2}=\frac{\left(\sum_{k=1}^{4}\left[A_{k}^{2} \operatorname{sh}\left(\beta \frac{k H}{2}\right)+B_{k}^{2} \operatorname{ch}\left(\beta \frac{k H}{2}\right)\right]\right)\left(\sum_{k=1}^{4}\left[A_{k}^{4} \operatorname{sh}\left(\beta \frac{k H}{2}\right)+B_{k}^{4} \operatorname{ch}\left(\beta \frac{k H}{2}\right)\right]\right)}{16 \sum_{k=0}^{2} D_{k} \operatorname{ch}(\beta k H)} e^{-\frac{r}{\xi}}, \\
& \left\langle S_{j, 1}^{z} S_{j+r, 2}^{z}\right\rangle-\left\langle S_{j, 1}^{z}\right\rangle\left\langle S_{j, 2}^{z}\right\rangle=\frac{\left(\sum_{k=1}^{4}\left[A_{k}^{1} \operatorname{sh}\left(\beta \frac{k H}{2}\right)+B_{k}^{1} \operatorname{ch}\left(\beta \frac{k H}{2}\right)\right]\right)\left(\sum_{k=1}^{4}\left[A_{k}^{4} \operatorname{sh}\left(\beta \frac{k H}{2}\right)+B_{k}^{4} \operatorname{ch}\left(\beta \frac{k H}{2}\right)\right]\right)}{16 \sum_{k=0}^{2} D_{k} \operatorname{ch}(\beta k H)} e^{-\frac{r}{\xi}}, \\
& \left\langle S_{j, 2}^{z} S_{j+r, 1}^{z}\right\rangle-\left\langle S_{j, 1}^{z}\right\rangle\left\langle S_{j, 2}^{z}\right\rangle=\frac{\left(\sum_{k=1}^{4}\left[A_{k}^{2} \operatorname{sh}\left(\beta \frac{k H}{2}\right)+B_{k}^{2} \operatorname{ch}\left(\beta \frac{k H}{2}\right)\right]\right)\left(\sum_{k=1}^{4}\left[A_{k}^{3} \operatorname{sh}\left(\beta \frac{k H}{2}\right)+B_{k}^{3} \operatorname{ch}\left(\beta \frac{k H}{2}\right)\right]\right)}{16 \sum_{k=0}^{2} D_{k} \operatorname{ch}(\beta k H)} e^{-\frac{r}{\xi}}, \\
& \left\langle S_{j, 1}^{z} \sigma_{j+r}\right\rangle-\left\langle S_{j, 1}^{z}\right\rangle\left\langle\sigma_{j}\right\rangle=-\frac{e^{-\beta \frac{J}{4}} \sqrt{1-M_{\sigma}^{2}} \sum_{k=1}^{4}\left(A_{k}^{1} \operatorname{sh}\left(\beta \frac{k H}{2}\right)+B_{k}^{1} \operatorname{ch}\left(\beta \frac{k H}{2}\right)\right)}{4 \lambda_{1}^{S C}} e^{-\frac{r}{\xi}}, \\
& \left\langle S_{j, 2}^{z} \sigma_{j+r}\right\rangle-\left\langle S_{j, 2}^{z}\right\rangle\left\langle\sigma_{j}\right\rangle=-\frac{e^{-\beta \frac{J}{4}} \sqrt{1-M_{\sigma}^{2}} \sum_{k=1}^{4}\left(A_{k}^{2} \operatorname{sh}\left(\beta \frac{k H}{2}\right)+B_{k}^{2} \operatorname{ch}\left(\beta \frac{k H}{2}\right)\right)}{4 \lambda_{1}^{S C}} e^{-\frac{r}{\xi}}, \\
& \left\langle S_{j, 1}^{z} \tau_{j+r}\right\rangle-\left\langle S_{j, 1}^{z}\right\rangle\left\langle\tau_{j}\right\rangle=\frac{\left(\sum_{k=1}^{4}\left[A_{k}^{1} \operatorname{sh}\left(\beta \frac{k H}{2}\right)+B_{k}^{1} \operatorname{ch}\left(\beta \frac{k H}{2}\right)\right]\right)\left(\sum_{k=0}^{4}\left[A_{k}^{6} \operatorname{sh}\left(\beta \frac{k H}{2}\right)+B_{k}^{6} \operatorname{ch}\left(\beta \frac{k H}{2}\right)\right]\right)}{16 \sum_{k=0}^{2} D_{k} \operatorname{ch}(\beta k H)} e^{-\frac{r}{\xi}}, \\
& \left\langle S_{j, 2}^{z} \tau_{j+r}\right\rangle-\left\langle S_{j, 2}^{z}\right\rangle\left\langle\tau_{j}\right\rangle=\frac{\left(\sum_{k=1}^{4}\left[A_{k}^{2} \operatorname{sh}\left(\beta \frac{k H}{2}\right)+B_{k}^{2} \operatorname{ch}\left(\beta \frac{k H}{2}\right)\right]\right)\left(\sum_{k=0}^{4}\left[A_{k}^{6} \operatorname{sh}\left(\beta \frac{k H}{2}\right)+B_{k}^{6} \operatorname{ch}\left(\beta \frac{k H}{2}\right)\right]\right)}{16 \sum_{k=0}^{2} D_{k} \operatorname{ch}(\beta k H)} e^{-\frac{r}{\xi}}, \\
& \left\langle\sigma_{j} S_{j+r, 1}^{z}\right\rangle-\left\langle S_{j, 1}^{z}\right\rangle\left\langle\sigma_{j}\right\rangle=-\frac{e^{-\beta \frac{J}{4}} \sqrt{1-M_{\sigma}^{2}} \sum_{k=1}^{4}\left(A_{k}^{3} \operatorname{sh}\left(\beta \frac{k H}{2}\right)+B_{k}^{3} \operatorname{ch}\left(\beta \frac{k H}{2}\right)\right)}{4 \lambda_{1}^{S C}} e^{-\frac{r}{\xi}}, \\
& \left\langle\sigma_{j} S_{j+r, 2}^{z}\right\rangle-\left\langle S_{j, 2}^{z}\right\rangle\left\langle\sigma_{j}\right\rangle=-\frac{e^{-\beta \frac{J}{4}} \sqrt{1-M_{\sigma}^{2}} \sum_{k=1}^{4}\left(A_{k}^{4} \operatorname{sh}\left(\beta \frac{k H}{2}\right)+B_{k}^{4} \operatorname{ch}\left(\beta \frac{k H}{2}\right)\right)}{4 \lambda_{1}^{S C}} e^{-\frac{r}{\xi}}, \\
& \left\langle\sigma_{j} \tau_{j+r}\right\rangle-\left\langle\sigma_{j}\right\rangle\left\langle\tau_{j}\right\rangle=-\frac{e^{-\beta \frac{J}{4}} \sqrt{1-M_{\sigma}^{2}} \sum_{k=0}^{4}\left[A_{k}^{6} \operatorname{sh}\left(\beta \frac{k H}{2}\right)+B_{k}^{6} \operatorname{ch}\left(\beta \frac{k H}{2}\right)\right]}{4 \lambda_{1}^{S C}} e^{-\frac{r}{\xi}}, \\
& \left\langle\tau_{j} S_{j+r, 1}^{z}\right\rangle-\left\langle S_{j, 1}^{z}\right\rangle\left\langle\tau_{j}\right\rangle=\frac{\left(\sum_{k=0}^{4}\left[A_{k}^{5} \operatorname{sh}\left(\beta \frac{k H}{2}\right)+B_{k}^{5} \operatorname{ch}\left(\beta \frac{k H}{2}\right)\right]\right)\left(\sum_{k=1}^{4}\left[A_{k}^{3} \operatorname{sh}\left(\beta \frac{k H}{2}\right)+B_{k}^{3} \operatorname{ch}\left(\beta \frac{k H}{2}\right)\right]\right)}{16 \sum_{k=0}^{2} D_{k} \operatorname{ch}(\beta k H)} e^{-\frac{r}{\xi}}, \\
& \left\langle\tau_{j} S_{j+r, 2}^{z}\right\rangle-\left\langle S_{j, 2}^{z}\right\rangle\left\langle\tau_{j}\right\rangle=\frac{\left(\sum_{k=0}^{4}\left[A_{k}^{5} \operatorname{sh}\left(\beta \frac{k H}{2}\right)+B_{k}^{5} \operatorname{ch}\left(\beta \frac{k H}{2}\right)\right]\right)\left(\sum_{k=1}^{4}\left[A_{k}^{4} \operatorname{sh}\left(\beta \frac{k H}{2}\right)+B_{k}^{4} \operatorname{ch}\left(\beta \frac{k H}{2}\right)\right]\right)}{16 \sum_{k=0}^{2} D_{k} \operatorname{ch}(\beta k H)} e^{-\frac{r}{\xi}}, \\
& \left\langle\tau_{j} \sigma_{j+r}\right\rangle-\left\langle\sigma_{j}\right\rangle\left\langle\tau_{j}\right\rangle=-\frac{e^{-\beta \frac{J}{4}} \sqrt{1-M_{\sigma}^{2}} \sum_{k=0}^{4}\left[A_{k}^{5} \operatorname{sh}\left(\beta \frac{k H}{2}\right)+B_{k}^{5} \operatorname{ch}\left(\beta \frac{k H}{2}\right)\right]}{4 \lambda_{1}^{S C}} e^{-\frac{r}{\xi}}, \\
& \left\langle\tau_{j} \tau_{j+r}\right\rangle-\left\langle\tau_{j}\right\rangle^{2}=\frac{\left(\sum_{k=0}^{4}\left[A_{k}^{5} \operatorname{sh}\left(\beta \frac{k H}{2}\right)+B_{k}^{5} \operatorname{ch}\left(\beta \frac{k H}{2}\right)\right]\right)\left(\sum_{k=0}^{4}\left[A_{k}^{6} \operatorname{sh}\left(\beta \frac{k H}{2}\right)+B_{k}^{6} \operatorname{ch}\left(\beta \frac{k H}{2}\right)\right]\right)}{16 \sum_{k=0}^{2} D_{k} \operatorname{ch}(\beta k H)} e^{-\frac{r}{\xi}},
\end{aligned}
$$


The coefficients in Eqs.4.14) and (4.15) are

$$
\begin{aligned}
& A_{1}^{1}=e^{\beta \frac{3 K}{4}}-e^{-\beta \frac{K}{4}}\left(1+2 W M_{\sigma}\right), \quad A_{2}^{1}=2 e^{\beta \frac{K}{4}} \operatorname{sh}\left(\beta \frac{K}{2}\right) \sqrt{1-M_{\sigma}^{2}}, \quad A_{3}^{1}=0, \quad A_{4}^{1}=-e^{-\beta \frac{5 K}{4}} \sqrt{1-M_{\sigma}^{2}}, \\
& B_{1}^{1}=\left(e^{\beta \frac{3 K}{4}}+e^{-\beta \frac{K}{4}}\right) M_{\sigma}-2 e^{-\beta \frac{K}{4}} W, \quad B_{2}^{1}=2 W e^{-\beta \frac{K}{4}} \sqrt{1-M_{\sigma}^{2}}, \quad B_{3}^{1}=2 e^{-\beta \frac{K}{4}} M_{\sigma}, \quad B_{4}^{1}=0, \\
& A_{1}^{2}=e^{\beta \frac{3 K}{4}}-e^{-\beta \frac{K}{4}}\left(1-2 W M_{\sigma}\right), \quad A_{2}^{2}=A_{2}^{1}, A_{3}^{2}=0, A_{4}^{2}=A_{4}^{1}, \\
& B_{1}^{2}=\left(e^{\beta \frac{3 K}{4}}+e^{-\beta \frac{K}{4}}\right) M_{\sigma}+2 e^{-\beta \frac{K}{4}} W, \quad B_{2}^{2}=-2 W e^{-\beta \frac{K}{4}} \sqrt{1-M_{\sigma}^{2}}, \quad B_{3}^{2}=B_{3}^{1}, \quad B_{4}^{2}=0, \\
& A_{1}^{3}=-e^{\beta \frac{3 K}{4}}+e^{-\beta \frac{K}{4}}\left(1-2 W M_{\sigma}\right), \quad A_{2}^{3}=A_{2}^{1}, A_{3}^{3}=0, \quad A_{4}^{3}=A_{4}^{1}, \\
& B_{1}^{3}=B_{1}^{2}, \quad B_{2}^{3}=B_{2}^{1}, \quad B_{3}^{3}=B_{2}^{1}, \quad B_{4}^{3}=0, \\
& A_{1}^{4}=-e^{\beta \frac{3 K}{4}}+e^{-\beta \frac{K}{4}}\left(1+2 W M_{\sigma}\right), \quad A_{2}^{4}=A_{2}^{1}, \quad A_{3}^{4}=0, \quad A_{4}^{4}=A_{4}^{1} \\
& B_{1}^{4}=B_{1}^{1}, \quad B_{2}^{4}=-B_{1}^{2}, \quad B_{3}^{4}=B_{3}^{1}, \quad B_{4}^{4}=0, \\
& A_{1}^{5}=\left(2 e^{-\beta \frac{K}{4}} Q_{2}^{S C}+2 e^{\beta \frac{K}{4}} Q_{1}^{S C}-e^{-\beta \frac{K}{4}}-e^{\beta \frac{3 K}{4}}\right) M_{\sigma}, A_{2}^{5}=0, A_{3}^{5}=2 e^{-\beta \frac{K}{4}} M_{\sigma}, A_{4}^{5}=0 \\
& B_{0}^{5}=\left(2 e^{\beta \frac{K}{4}} Q_{1}^{S C}-e^{\beta \frac{3 K}{4}}\right) \sqrt{1-M_{\sigma}^{2}}, \quad B_{1}^{5}=e^{-\beta \frac{K}{4}}\left(1+2 Q_{2}^{S C}\right)-2 e^{\beta \frac{K}{4}}-e^{\beta \frac{3 K}{4}}, \\
& B_{2}^{5}=2\left(e^{\beta \frac{K}{4}} \operatorname{ch}\left(\beta \frac{K}{2}\right)-e^{-\beta \frac{K}{4}} Q_{2}^{S C}\right) \sqrt{1-M_{\sigma}^{2}}, B_{3}^{5}=0, B_{4}^{5}=-e^{-\beta \frac{5 K}{4}} \sqrt{1-M_{\sigma}^{2}} \\
& A_{1}^{6}=A_{1}^{5}, \quad A_{2}^{6}=0, \quad A_{3}^{6}=A_{3}^{5}, \quad A_{4}^{6}=0 \text {, } \\
& B_{0}^{6}=B_{0}^{5}, \quad B_{1}^{6}=-B_{1}^{5}, \quad B_{2}^{6}=B_{2}^{5}, \quad B_{3}^{6}=0, B_{4}^{6}=B_{4}^{5} \text {, } \\
& D_{0}=e^{-\beta \frac{K}{2}} \operatorname{sh}^{2}(\beta K)+\left(e^{\beta K}-1\right) Q_{1}^{S C}-2 Q_{2}^{S C} \operatorname{sh}\left(\beta \frac{K}{2}\right) \text {, } \\
& D_{1}=2 e^{-\beta \frac{K}{2}}(\operatorname{ch}(\beta K)-1) Q_{2}^{S C}+\operatorname{ch}\left(\beta \frac{3 K}{2}\right)-\operatorname{ch}\left(\beta \frac{K}{2}\right), D_{2}=2 Q_{2}^{S C} \operatorname{sh}\left(\beta \frac{K}{2}\right)+\left(e^{-\beta K}-1\right) Q_{1}^{S C}, \\
& S_{0}=e^{\beta \frac{3 K}{4}}+2 e^{\beta \frac{K}{4}} Q_{1}^{S C}, \quad S_{1}=2\left(e^{-\beta \frac{K}{4}} Q_{2}^{S C}+e^{\beta \frac{K}{4}} \operatorname{ch}\left(\beta \frac{K}{2}\right)\right), \quad S_{2}=e^{-\beta \frac{5 K}{4}} .
\end{aligned}
$$

To illustrate the spatial and temperature dependence of various correlation functions of Eq.(4.14) it is helpful to look at their plots. First of all, the general remarks about correlations in HIC are noteworthy. The general structure of the HIC exclude any possibility to have an entangled non trivial macroscopic ground state, on the contrary, any macroscopic ground state is just a tensor product of the local ground states, which, in their turn, could be entangled. Thus, the zero-temperature long-range correlations between the spins from different blocks, calculated with respect to their expectation values, are trivial which implies the zero value of all correlation function from Eq. (4.14) at $T=0$, as for the arbitrary ground state $|\Psi\rangle=\prod_{i=1}^{N}\left|\psi_{i}\right\rangle$ one has

$$
\begin{aligned}
& \left\langle\Psi\left|P_{j} R_{j+r}\right| \Psi\right\rangle=\left\langle\psi_{j}\left|P_{j}\right| \psi_{j}\right\rangle\left\langle\psi_{j+r}\left|R_{j+1}\right| \psi_{j+r}\right\rangle= \\
& \left\langle\Psi\left|P_{j}\right| \Psi\right\rangle\left\langle\Psi\left|R_{j+r}\right| \Psi\right\rangle .
\end{aligned}
$$

However, the thermal fluctuations as well as the effect of the magnetic field lead to non-zero correlations within a narrow interval of low temperatures for short distances. The plots of the temperature dependence of all corre- lation functions for the asymmetric sawtooth chain are presented in Figure 3 for the distance equal to one block, $r=1$. Here, the values of the parameters correspond to the spin-modulated zero-temperature ground state of the asymmetric Heisenberg-Ising sawtooth chain ${ }^{10,13}$. It is rather expectable that the finite-temperature correlations are mostly of short range with respect to blocks, but as one block contains four spins, the non-zero, though weak, correlations even for $r=1$ are the non-trivial result. One can see from the plots in Figure 3 the vanishing of all correlations at high as well as at low temperatures as was mentioned above. As expected, the closer to each other are situated the spins from different blocks, the larger is the magnitude of the correlation between them. For instance, the correlation between $\tau_{j}$ and $S_{j+1,1}^{z}$ at the temperature corresponding to the peak is four times larger in absolute value than the correlation between $\tau_{j}$ and $S_{j+1,2}^{z}$ and more that seventy times larger than the correlation between $S_{j, 2}^{z}$ and $\tau_{j+1}$. In the same time, the correlation between $S_{j, 1}^{z}$ are the strongest ones, though, the are separated by The dependence of the correlation functions against the distance for the same values of cou- 
pling constants and anisotropy and for temperatures corresponding to the strongest correlation of the thermal fluctuations are presented in Figure 4. For all correlation functions one can see the usual exponential decay in their absolute value. The sign of the correlations is defined by the mutual orientation of the corresponding spins which can be seen in the $T=0$ ground state ${ }^{10,13}$ for the given set of the parameters. In Figure 5 we presented the plots of the same correlation functions $\left\langle S_{j, 1}^{z} \tau_{j+r}\right\rangle-\left\langle S_{j, 1}^{z}\right\rangle\left\langle\tau_{j}\right\rangle$ and $\left\langle\tau_{j} S_{j+r, 1}^{z}\right\rangle-\left\langle S_{j, 1}^{z}\right\rangle\left\langle\tau_{j}\right\rangle$ against the distance for different temperatures. The non-monotonous behavior of the correlation functions described above can be clearly seen from the plots.

\section{CONCLUSION}

In the present paper we elaborated a technique for calculating correlation functions in HIC. The technique is based on the classical transfer-matrix formalism which proves itself as the most efficient and straightforward method of treating the HIC, allowing one to obtain exact expressions for the partition function and, thus, for all thermodynamic functions in a quite simple way $5,9-11,13-17,19-22,26$. However, the classical transfermatrix formalism is also very convenient for the calculation of the correlation functions of various kinds, because all canonical expectation values are expressed in terms of traces of certain products of the transfer-matrices with matrices representing the microscopic variables in the transfer-matrix formalism ${ }^{27}$. We presented a detailed scheme of calculation of the correlation functions for HIC, obtained general expressions for the corresponding trace formulas and presented the general expressions for the matrix representations of the main microscopic variables for the typical HIC, quantum spin operators, additional classical spins from block Hamiltonians and intermediate spin. As it is expected from general principles, the spatial decay of any correlation between any pair of spin is characterized by a single correlation length obtained in a standard way as the reciprocal of the logarithm of the ratio of the largest and second largest eigenvalues of the classical transfer matrix. In the simplest case considered here when intermediate classical $\sigma$-spins are $1 / 2$ spins the transfer matrix has dimension 2 by 2 and, thus, only two eigenvalues. However, the prefactors are strongly affected by the microscopic variables. We illustrated our general formalism by two examples, the simplest HIC, symmetric Heisenberg-Ising diamond-chain and asymmetric Heisenberg-Ising sawtooth chain with a pair of quantum spins in the unit cell. We presented the exact form of the transfer-matrix, its eigenvalues, matrix representation corresponding to the classical transfer-matrix formalism for quantum spin operators, matrix representation for additional classical spin from block Hamiltonian and all possible pair correlation functions. A very important feature of the correlations function of the HIC considered in the present paper is the absence of correlations between transverse components of quantum spins from different blocks. The corresponding matrix representation of the $S_{n, 1}^{x}$ and $S_{n, a}^{y}$ operators are identically zero. This fact is an obvious consequence of the structure of the HIC where blocks interact to each other only through $z$-components of the spins.

It is also worth mentioning that the general idea of using the classical transfer-matrix for describing the thermodynamic properties of decorated spin lattices can be applied also for two-dimensional systems. Although the existing examples of the papers devoted to the topic deal with the decoration-iteration transformation ${ }^{38,40,43-46}$, the direct generalization of the existing transfer-matrix method is possible. As known, two-dimensional systems can exhibit phase transitions at finite temperature in contrast to the one-dimensional models where only zero-temperature transitions are possible. In principle, two-dimensional spin systems can possess a complicated structure of the order parameters, and multiple orderings, which, particularly, can be different in different spatial directions. For these reasons, the statement about the unique correlation length is no longer valid here.

\section{ACKNOWLEDGEMENTS}

V.O. expresses his gratitude to the LNF-INFN in Frascati for warm hospitality during the work on the paper. He also acknowledges partial financial support from Volkswagen Foundation (Grant No. I/84 496) and from the project SCS-BFBR 11RB-001.
1 J. Strečka and M. Jaščur, J. Phys.: Condens. Matter 15, 4519 (2003).

2 J. Strečka, M. Hagiwara, M. Jaščur, and K. Minami, Czech. J. Phys. 54, D583 (2004).

3 J. Strečka, M. Jaščur, M. Hagiwara, Y. Narumi, and K. Kindo, K. Minami, Phys. Rev. B 72, 024459 (2005).

${ }^{4}$ L. Čanová, J. Strečka and M. Jaščur, J. Phys. : Condens. Matter 18, 4967 (2006).

5 J. S. Valverde, O. Rojas, and S. M. de Souza, J. Phys.: Condens. Matter 20, 345208 (2008).
${ }^{6}$ M. S. S. Pereira, F. A. B. F. de Moura, and M. L. Lyra, Phys. Rev. B 77, 024402 (2008).

7 M. S. S. Pereira, F. A. B. F. de Moura, and M. L. Lyra, Phys. Rev. B 79, 054427 (2009).

8 J. Strečka, L. Čanová, T. Lučivjanský, and M. Jaščur, J. Phys.: Conf. Ser. 145, 012058 (2009).

9 D. Antonosyan, S. Bellucci, and V. Ohanyan, Phys. Rev. B 79, 014432 (2009).

10 V. Ohanyan, Condens. Matter Phys. 12, 343 (2009).

11 V. Ohanyan, Phys. Atomic Nucl. 73, 494 (2010). 

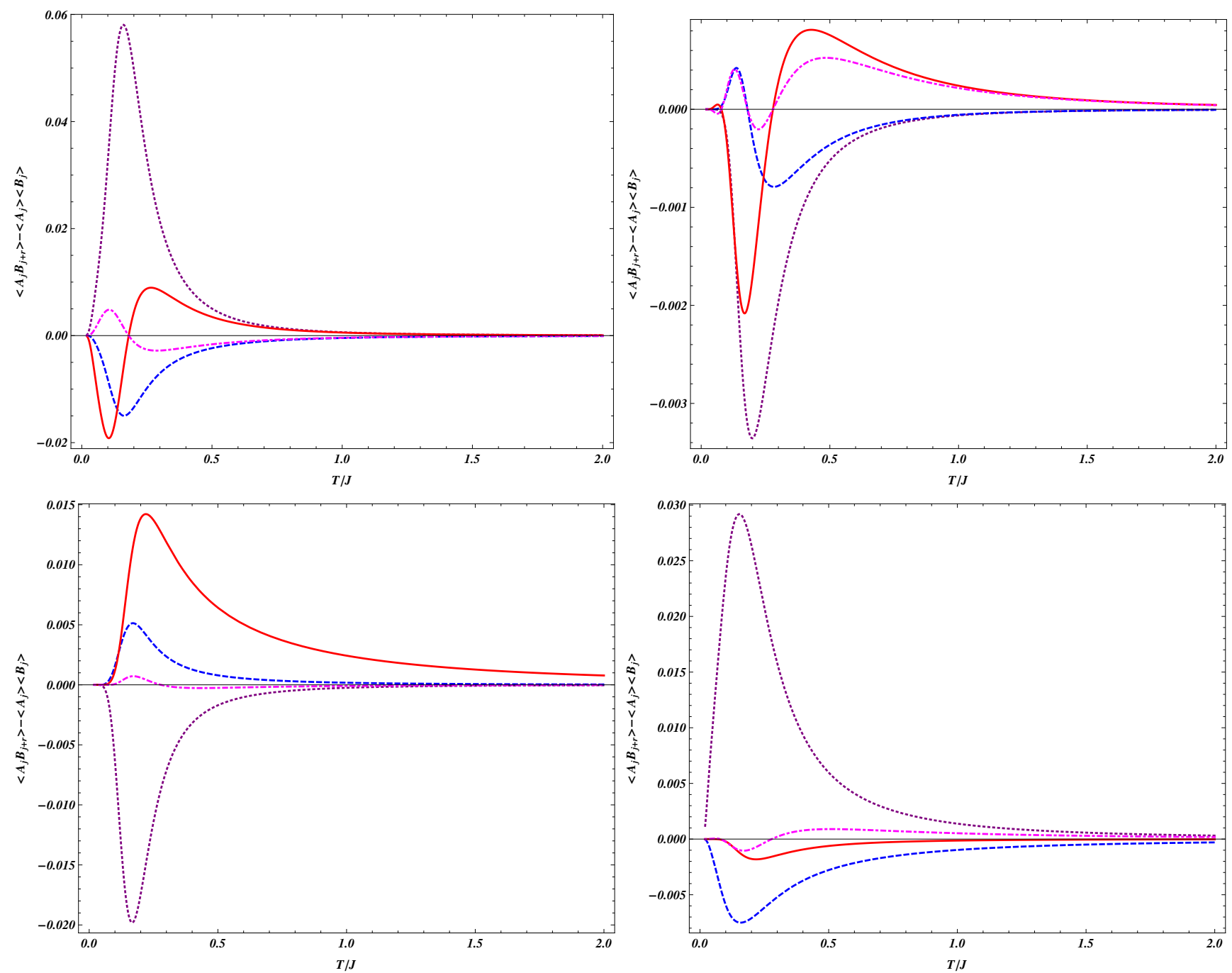

FIG. 3: (Color online) The temperature dependence of the correlation functions for the asymmetric sawtooth chain for $K / J=$ $0.5, H / J=0.5, \Delta=0.3$ at $r=1$ (See Eq. 4.14). Upper left panel: $\left\langle S_{j, 1}^{z} S_{j+r, 1}^{z}\right\rangle-\left\langle S_{j, 1}^{z}\right\rangle^{2}$ - dotted (purple); $\left\langle S_{j, 1}^{z} S_{j+r, 2}^{z}\right\rangle-$ $\left\langle S_{j, 1}^{z}\right\rangle\left\langle S_{j, 2}^{z}\right\rangle$ - dashed (blue); $\left\langle S_{j, 2}^{z} S_{j+r, 1}^{z}\right\rangle-\left\langle S_{j, 1}^{z}\right\rangle\left\langle S_{j, 2}^{z}\right\rangle$ - solid (red); $\left\langle S_{j, 2}^{z} S_{j+r, 2}^{z}\right\rangle-\left\langle S_{j, 2}^{z}\right\rangle^{2}$ - dot-dashed (magenta). Upper right panel: $\left\langle S_{j, 1}^{z} \sigma_{j+r}\right\rangle-\left\langle S_{j, 1}^{z}\right\rangle\left\langle\sigma_{j}\right\rangle$ - dotted (purple); $\left\langle S_{j, 2}^{z} \sigma_{j+r}\right\rangle-\left\langle S_{j, 2}^{z}\right\rangle\left\langle\sigma_{j}\right\rangle$ - dashed (blue); $\left\langle S_{j, 1}^{z} \tau_{j+r}\right\rangle-\left\langle S_{j, 1}^{z}\right\rangle\left\langle\tau_{j}\right\rangle-$ solid (red); $\left\langle S_{j, 2}^{z} \tau_{j+r}\right\rangle-\left\langle S_{j, 2}^{z}\right\rangle\left\langle\tau_{j}\right\rangle$ - dot dashed (magenta). Lower left panel: $\left\langle\sigma_{j} S_{j+r, 1}^{z}\right\rangle-\left\langle S_{j, 1}^{z}\right\rangle\left\langle\sigma_{j}\right\rangle-\operatorname{dotted}\left(\right.$ purple), $\left\langle\sigma_{j} S_{j+r, 2}^{z}\right\rangle-\left\langle S_{j, 2}^{z}\right\rangle\left\langle\sigma_{j}\right\rangle$ - dashed (blue), $\left\langle\sigma_{j} \sigma_{j+r, 1}^{z}\right\rangle-\left\langle\sigma_{j}\right\rangle^{2}$ - solid (red); $\left\langle\sigma_{j} \tau_{j+r, 1}^{z}\right\rangle-\left\langle\sigma_{j}\right\rangle\left\langle\tau_{j}\right\rangle$ - dot-dashed (magenta). Lower right panel: $\left\langle\tau_{j} S_{j+r, 1}^{z}\right\rangle-$ $\left\langle S_{j, 1}^{z}\right\rangle\left\langle\tau_{j}\right\rangle$ - dotted (purple); $\left\langle\tau_{j} S_{j+r, 2}^{z}\right\rangle-\left\langle S_{j, 2}^{z}\right\rangle\left\langle\tau_{j}\right\rangle$ - dashed (blue); $\left\langle\tau_{j} \sigma_{j+r}^{z}\right\rangle-\left\langle\sigma_{j}\right\rangle\left\langle\tau_{j}\right\rangle$ - solid (red); $\left\langle\tau_{j} \tau_{j+r}\right\rangle-\left\langle\tau_{j}\right\rangle^{2}-$ dot-dashed (magenta).

12 J. Strečka, L. Galisova, and O. Derzhko, Acta Phys. Pol. A 118, 742 (2010).

13 S. Bellucci and V. Ohanyan, Eur. Phys. J. B 75, 531 (2010).

14 O. Rojas, S. M. de Souza, V. Ohanyan, and M. Khurshudyan, Phys. Rev. B 83, 094430 (2011).

15 O. Rojas and S. M. de Souza, Phys. Lett. A 375, 1295 (2011).

16 B. Lisnii, Low Temp. Phys. 37, 296 (2011).

17 B. Lisnii, Ukr. Journal of Phys. 56, 1237 (2011).

18 J. Strečka, M.Dančo, Physica B 406, 2967 (2011).

19 V. Ohanyan and A. Honecker, Phys. Rev. B 86, 054412 (2012).

20 L. Chakhmakhchyan, N. Ananikian, L. Ananikyan, and
Č. Burdık, J. Phys.: Conf. Ser. 343, 012022 (2012).

21 O. Rojas, S. M. de Souza, and N. S. Ananikian, Phys. Rev. E 85, 061123 (2012).

22 N. Ananikian, L. Ananikyan, L. Chakhmakhchyan, and O. Rojas, J. Phys.: Condens. Matter 24, 256001 (2012).

23 T. Verkholyak, J. Strečka, J.Phys. A: Mathematical and Theoretical, 45, 305001 (2012).

24 J. Strečka, M. Hagiwara, Y. Han, T. Kida, Z. Honda, and M. Ikeda, Condens. Matter Phys. 15, 43002 (2012).

25 L. Galisova, phys. stat. solidi(b) 250, 187 (2013).

26 O. Rojas, M. Rojas, N. S. Ananikian and S. M. de Souza, Phys. Rev. A 86, 042330 (2012).

27 R. Baxter, Exactly Solved Models in Statistical Mechanics, (Academic Press, New York, 1982). 

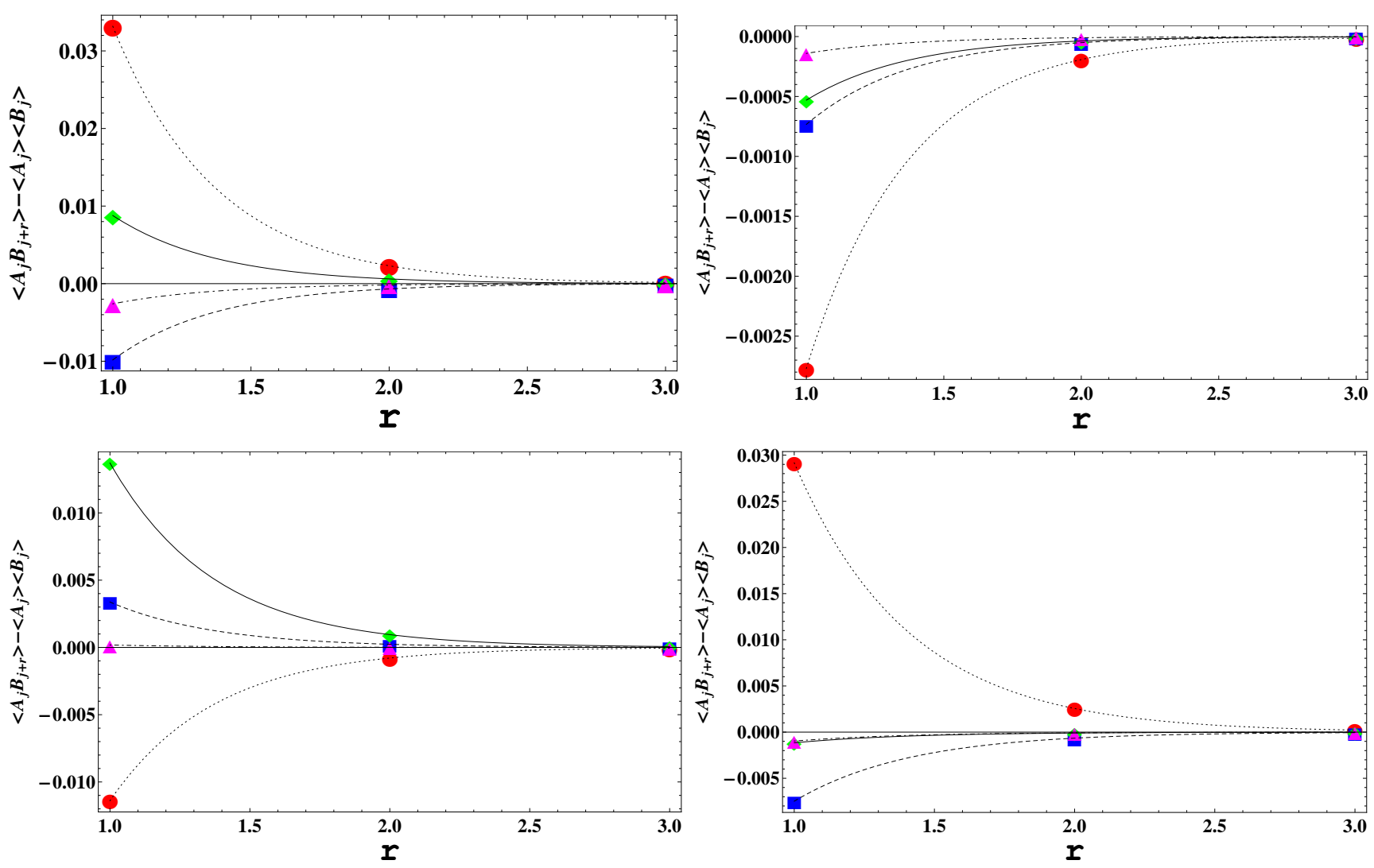

FIG. 4: (Color online) The dependence of the correlation functions for the asymmetric sawtooth chain against the distance for $K / J=0.5, H / J=0.5, \Delta=0.3$ at $r=1,2$ and 3 (See Eq. 4.14) The lines are just the guides for the eyes. Upper left panel: $T / J=0.25,\left\langle S_{j, 1}^{z} S_{j+r, 1}^{z}\right\rangle-\left\langle S_{j, 1}^{z}\right\rangle^{2}$ - red circles (dotted); $\left\langle S_{j, 1}^{z} S_{j+r, 2}^{z}\right\rangle-\left\langle S_{j, 1}^{z}\right\rangle\left\langle S_{j, 2}^{z}\right\rangle$ - blue squares (dashed); $\left\langle S_{j, 2}^{z} S_{j+r, 1}^{z}\right\rangle-$ $\left\langle S_{j, 1}^{z}\right\rangle\left\langle S_{j, 2}^{z}\right\rangle$ - green diamonds (solid); $\left\langle S_{j, 2}^{z} S_{j+r, 2}^{z}\right\rangle-\left\langle S_{j, 2}^{z}\right\rangle^{2}$ - magenta triangles (dot-dashed). Upper right panel: $T / J=0.25$, $\left\langle S_{j, 1}^{z} \sigma_{j+r}\right\rangle-\left\langle S_{j, 1}^{z}\right\rangle\left\langle\sigma_{j}\right\rangle$ - red circles (dotted); $\left\langle S_{j, 2}^{z} \sigma_{j+r}\right\rangle-\left\langle S_{j, 2}^{z}\right\rangle\left\langle\sigma_{j}\right\rangle$ - blue squares (dashed); $\left\langle S_{j, 1}^{z} \tau_{j+r}\right\rangle-\left\langle S_{j, 1}^{z}\right\rangle\left\langle\tau_{j}\right\rangle-$ green diamonds (solid); $\left\langle S_{j, 2}^{z} \tau_{j+r}\right\rangle-\left\langle S_{j, 2}^{z}\right\rangle\left\langle\tau_{j}\right\rangle$ - magenta triangles (dot-dashed). Lower left panel: $T / J=0.25,\left\langle\sigma_{j} S_{j+r, 1}^{z}\right\rangle-\left\langle S_{j, 1}^{z}\right\rangle\left\langle\sigma_{j}\right\rangle$ - red circles (dotted), $\left\langle\sigma_{j} S_{j+r, 2}^{z}\right\rangle-\left\langle S_{j, 2}^{z}\right\rangle\left\langle\sigma_{j}\right\rangle$ - blue squares (dashed), $\left\langle\sigma_{j} \sigma_{j+r, 1}\right\rangle-\left\langle\sigma_{j}\right\rangle^{2}$ - green diamonds (solid); $\left\langle\sigma_{j} \tau_{j+r, 1}\right\rangle-$ $\left\langle\sigma_{j}\right\rangle\left\langle\tau_{j}\right\rangle$ - magenta triangles (dot-dashed). Lower right panel: $T / J=0.15,\left\langle\tau_{j} S_{j+r, 1}^{z}\right\rangle-\left\langle S_{j, 1}^{z}\right\rangle\left\langle\tau_{j}\right\rangle$ - red circles $($ dotted); $\left\langle\tau_{j} S_{j+r, 2}^{z}\right\rangle-\left\langle S_{j, 2}^{z}\right\rangle\left\langle\tau_{j}\right\rangle$ - blue squares (dashed); $\left\langle\tau_{j} \sigma_{j+r}\right\rangle-\left\langle\sigma_{j}\right\rangle\left\langle\tau_{j}\right\rangle$ - green diamonds (solid); $\left\langle\tau_{j} \tau_{j+r}\right\rangle-\left\langle\tau_{j}\right\rangle^{2}-$ magenta triangles (dot-dashed).


FIG. 5: (Color online) The dependence of the correlation functions $\left\langle S_{j, 1}^{z} \tau_{j+r}\right\rangle-\left\langle S_{j, 1}^{z}\right\rangle\left\langle\tau_{j}\right\rangle$ (left panel) and $\left\langle\tau_{j} S_{j+r, 1}^{z}\right\rangle-\left\langle S_{j, 1}^{z}\right\rangle\left\langle\tau_{j}\right\rangle$ (right panel) against the distance for different temperatures at $r=1,2$ and 3 . The lines are just the guides for the eyes. $T / J=0.05$ - red bullets (dot-dashed ) $T / J=0.18$ - blue squares (dashed) $T / J=0.28$ - green diamonds $($ dotted) $; T / J=0.42$ - magenta triangles (solid). 
28 V. Ohanyan and N. Ananikian, Phys. Lett. A 307, 76 (2003).

29 E. Aydiner and C. Akyüz, Chin. Phys. Lett. 22, 2382 (2005).

30 E. Aydiner, C. Akyüz, M. Gönülol, and H. Polat, phys. stat. sol(b) 243, 2901 (2006).

31 D. Gatteschi, R. Sessoli, and J. Villain, Molecular Nanomagnets (Oxford Univ. PRess, New York 2007).

32 D. Visinescu, A. M. Madalan, M. Andruh, C. Duhayon, J.-P. Sutter, L. Ungur, W. Van den Heuvel, and L. F. Chibotaru, Chem. Eur. J. 15, 11808 (2009).

33 J. Rinck, G. Novitchi, W. Van den Heuvel, L. Ungur, Y. Lan, W. Wernsdorfer, C. E. Anson, L. F. Chibotaru, and A. K. Powell, Angew. Chem., Int. Ed. 49, 7583 (2010).

${ }^{34}$ W. Van den Heuvel and L. F. Chibotaru, Phys. Rev. B 82, 174436 (2010).

35 V. Ravi Chandra, S. Ramasesha, and D. Sen, Phys. Rev. B 70, 144404 (2004).

36 T. S. Venkatakrishnan, S. Sahoo, Nicolas Bréfuel, C. Duhayon, C. Paulsen, A.-L. Barra, S. Ramasesha, and J.-
P. Sutter, J. Am. Chem. Soc. 132, 6047 (2010).

37 S. Sahoo, J.-P. Sutter, and S. Ramasesha, J. Stat. Phys. 147, 181 (2012).

38 M. E. Fisher, Phys. Rev. 113, 969 (1959).

39 O. Rojas, J. S. Valverde and S. M. de Souza, Physica A 388, 1419 (2009).

40 J. Strečka, Phys. Lett. A 374, 3718 (2010).

41 M. Knežević and S. Milošević, J. Phys. A: Math. Gen. 13, 2479 (1980).

42 R. J. V. dos Santos and M. L. Lyra, Physica A 182, 133 (1992).

43 J. Strečka, L. Čanová, and K. Minami, Phys. Rev. B 79, 051103 (2009).

44 J. Strečka, O. Rojas, and S. M. de Souza, Eur. Phys. J. B 85, 54 (2010).

45 J.Čiśarova, and J. Strečka, Phys. Rev. B 87, 024421 (2013).

46 J.Čiśarova, F. Michaud, F. Mila, and J. Strečka, Phys. Rev. B 87, 054419 (2013). 